\title{
Diagnostic accuracy of Xpert Xpress Flu/RSV for detection of Influenza and Respiratory syncytial virus: systematic review and meta-analysis
}

\section{Xu-Guang Guo ( $\sim$ gysygxg@gmail.com )}

Department of Clinical Laboratory Medicine, The Third Affiliated Hospital of Guangzhou Medical University https://orcid.org/0000-0003-1302-5234

\section{Ye-Ling Liu}

Department of Clinical Medicine, The Third Clinical School of Guangzhou Medical University

\section{Tian-Ao Xie}

Deparement of Clinical Medicine,The Third Clinical School of Guangzhou Medical University

\section{Geng-Ling Lin}

Department of Clinical Medicine, The Third Clinical School of Guangzhou Medical University

\section{Qin-Rong Lin}

Department of Clinical Medicine, The Third Clinical School of Guangzhou Mediclal University

\section{Shu-Jin Fan}

Department of Clinical Medicine, The Third Clinical School of Guangzhou Medical University

\section{Zhen-Xing Li}

Department of Respiratory Medicine, The Third Affiliated Hospital of Guangzhou Medical University

\section{Shi Ouyang}

Department of Infectious disease, The Fifth Affiliated Hospital of Guangzhou Medical University

\section{Guo-Dong Zhu}

The Second Affiliated Hospital of South China University of Technology, Geriatrics related fundamental and clinical research

\section{Tian-Xing Ji}

Deparement of Clinical Medicine, The Second Affiliated Hospital of Guangzhou Medical University

\section{Zhi-Yong Pan}

Department of Clinical Medicine The Third Clinical School of Guangzhou Medical University

\section{Yong Xia}

Deparement of Clinical Laboratory Medicine, The Third Affiliated Hospital of Guangzhou Medical University

\section{Li-Juan Wu}

Baoan Matemal and Child Health Hospital of Jinan University 
Keywords: Xpert Xpress Flu/RSV, influenza virus,respiratory syncytial virus, PCR

Posted Date: December 1st, 2019

DOI: https://doi.org/10.21203/rs.2.17887/v1

License: (c) (1) This work is licensed under a Creative Commons Attribution 4.0 International License. Read Full License

Version of Record: A version of this preprint was published at Japanese Journal of Infectious Diseases on May 31st, 2021. See the published version at https://doi.org/10.7883/yoken.JJID.2020.987. 


\section{Abstract}

Background: Xpert Xpress Flu/RSV assay is a fast and automated real-time nucleic acid amplification method, recently approved by the USA, Europe and China for detection and differentiation of influenza (Flu) A, Flu B and RSV virus from nasopharyngeal swabs. Nevertheless, there are still no systematic reviews and meta-analysis on the accuracy of identification of influenza virus by The Xpert Xpress Flu/RSV assay. The aim of this meta-analysis was to verify the accuracy of Xpert Xpress Flu/RSV for detecting Flu and RSV.

Materials and Methods: The PubMed, EMBASE, Cochrane Library, and Web of Science databases were searched using the following keywords: Xpert Xpress Flu/RSV, Flu and RSV up to April 2019. Four researchers screened and extracted documents according to inclusion and exclusion criteria. The quality of original research was assessed using the QUADAS-2 guidelines. Meta-DiSc 1.4 software was used to analyze the sensitivity, specificity, positive likelihood ratio (+LR), negative likelihood ratio (-LR), diagnostic ratio (DOR), and Summary receiver operating characteristic curves (SROC). Deek's funnel plot asymmetry test was used to evaluate the publication bias by Stata 12.0 .

Results: Nine studies with 22 fourfold tables were included in this meta-analysis. The sensitivity of Xpert Xpress Flu/RSV for detection of Flu A, Flu B, and RSV was $0.96,0.98,0.98$; the specificity was $0.97,0.99$, 0.99; +LR was 80.65, 163.74, 237.35; -LR was 0.02 , 0.03, 0.05هDOR was 4806.90, 6266.78, 5254.41, respectively. AUC was all over 0.9. In addition, no publication bias for FluA, Flu B, and RSV was found.

Conclusions: Compared to the traditional PCR approach, Xpert Xpress Flu/RSV resulted as a valuable method for Flu and RSV diagnosis in the clinic, offering high sensitivity and specificity. Nonetheless, further research is necessary to determine whether Xpert Xpress Flu/RSV can be used as a clinical diagnostic standard for the identification of Flu and RSV. Keywords: Xpert Xpress Flu/RSV; influenza virus; respiratory syncytial virus; PCR

\section{Introduction}

Influenza is an acute infectious disease caused by a member of the orthomyxovirus family, which can be divided into two types: Flu $A$ and Flu $B^{1}$. Influenza can spread and cause seasonal epidemics all over the world, and in some extreme cases can even lead to death. Influenza viruses usually cause acute respiratory infections, pneumonia with acute respiratory distress development syndrome (ARDS) and respiratory failure ${ }^{1,2}$. Like influenza viruses, Respiratory syncytial virus (RSV) is another type of singlestrand RNA virus, which can affect all age groups ${ }^{3}$; however, children and elderly are more susceptible to RSV. In addition, it is the main cause of lower respiratory tract infection and hospitalization in children

under five years old, and the main cause of illness and death in the elderly ${ }^{3-5}$. Yet, the diagnosis of influenza virus and RSV in the respiratory tract infections is very difficult and requires molecular testing ${ }^{2,6}$. 
Over the last decade, rapid polymerase chain reaction (PCR) has been used to detect viruses affecting the respiratory tract ${ }^{7-9}$. Nevertheless, this method is time consuming and requires professional laboratories and specialized instruments. In addition, many intermediate steps of PCR experiments need to be performed by professionals, which may cause errors ${ }^{7,8,10}$. Under ideal circumstances, it should be possible to diagnose influenza should in a convenient and fast way without compromising accuracy ${ }^{11}$.

The Xpert Xpress Flu/RSV assay (Cepheid, Sunnyvale, CA) is a fast and automated real-time nucleic acid amplification test, which can be used for accurate and reliable detection of influenza $A$ (FluA), influenza $B$ (FluB) and RSV ${ }^{12,13}$. Compared to traditional PCR diagnosis assay, this method is very simple and can be used by everybody (do not require trained personnel) ${ }^{12}$. All the steps in this assay, including sample extraction, nucleic acid purification, and concentration, quantitative PCR amplification detection and analysis are completed automatically, which in turn significantly decreases the possibility of errors and time for diagnosis. In addition, this approach does not require precision pipetting ${ }^{10,12}$.

The Xpert Xpress Flu/RSV is an updated version of previous Xpert Flu/RSV XC, which requires less time (reduced the time to results (32 $\mathrm{min} .63 \mathrm{~min})$ ) and has higher sensitivity and specificity against RNA targets (including influenza viruses) ${ }^{14}$. In 2016, the use of Xpert Xpress Flu/RSV for in vitro diagnosis of FluA, FluB and RSV infection was approved by the US Food and Drug Administration (FDA) and European Medicines Agency (EMA), followed by National Medical Products Administration (NMPA) in China in $2019^{10}$. However, there are still no systematic reviews and meta-analysis on the accuracy of identification of influenza virus using The Xpert Xpress Flu/RSV assay. The aim of this meta-analysis was to verify the accuracy of Xpert Xpress Flu/RSV in detecting Flu and RSV. Our data provide new ideas and methods for clinical guidelines, and new means and gold standard for clinical diagnosis of influenza virus.

\section{Materials And Methods}

\section{Search for strategy and sources of information}

We respectively searched "Influenza" and "respiratory syncytial virus" in Medical Subject Headings terms (MeSH) and EMTREE terms as a supplement of synonyms. EMBASE, Cochrane Library, PubMed, Web of science were all searched from inception to April 2019, using the following keywords: "(Xpert AND (Influenza [all synonyms] OR RSV [all synonyms])). The reference sections of relevant articles were reviewed to find additional primary studies ${ }^{15}$. Finally, 46 articles in PubMed, 80 in Embase, 62 in Web of Science and 1 in Cochrane Library were found. Our study was carried out in March 2019.

\section{Inclusion and exclusion criteria}


The inclusion criteria were the following: (I) analysis of human specimens; (II) comparison of Xpert Xpress Flu/RSV with another gold standard (PCR) to detect the diagnostic accuracy of Flu/RSV; (III) data in the article were sufficient to draw a 2.2 table; (IV) articles published up to April 2019; (V) articles published in English; (VI) articles including RSV OR Influenza and Xpert.

The exclusion criteria were: (I) samples from animal or other species; (II) the gold standard was not revealed, or the Xpert Xpress Flu/RSV assay was not performed; (III) repeated Publications; (IV) conferences, letters, editorials, and abstracts.

\section{Research Screening and Selection}

The search results were imported into the Endnote X9. The two reviewers independently screened articles in accordance with inclusion and exclusion criteria. Duplicate references, conference abstracts, and unrelated articles were excluded. Subsequently, the full text of all selected studies was reviewed, quality assessed and the data were extracted. If there were disagreements, both reviewers would seek advice of a third reviewer.

\section{Data extraction and quality estimate}

Two reviewers extracted data independently from each study and resolved differences by consensus. Retrieved data included the year of publication, country, author, the number of samples, type of study (prospective or retrospective), the sample type in the article, gold standard, the diagnostic $2 \cdot 2$ table with its four cells: true positives, false negatives, true negatives and false positives. The methodological quality of each study was evaluated using the Quality Assessment of Diagnostic Accuracy Studies (QUADAS-2) tool of the diagnostic accuracy study ${ }^{16}$.

\section{Statistical analysis}

Two reviewers independently analyzed the data recommended for meta-analysis of the diagnostic studies through the meta-DiSc 1.4 software: specificity, sensitivity, negative likelihood ratio (-LR), positive likelihood ratio (+LR), diagnostic odds ratio (DOR). Summary of receiver operating characteristic curves (SROC) and the area under the curve (AUC) were used to summarize overall diagnostic performance. Deek's funnel plots asymmetry test was used to evaluate the publication bias by Stata12.0.

\section{Results}

\section{Studies characteristics}


After manually eliminating searched and duplicated articles, the title and main points from 93 articles were included in the final analysis. Seventy-six articles were excluded by screening title and abstract and 17 articles were retained for full-text scanning. In addition, 8 studies, including, five conference abstracts, two studies (not possible to extract a $2 \cdot 2$ table), and one letter article were additionally excluded.

Finally, nine articles including 4626 Influenza A samples, 4414 Influenza B samples and 3662 RSV samples were included in the meta-analysis $8,10,12-14,17-20$. The PRISMA flow diagram of this study is shown in Figure 1, while the study characteristics are shown in Table 1.

\section{QUADAS-2 quality evaluation results}

The quality of these nine articles were evaluated using QUADAS-2 software, and three evaluation criteria ("yes" represents "low" risk of deviation; "no" represents high risk of deviation, while "unclear" represents that the risk of deviation which is not well established). Specific quality evaluation results of included articles are shown in Table 2.

\section{Influenza A}

Nine reports evaluated Influenza A (Figure 2) $8,10,12-14,17-20$. The combined sensitivity, specificity, +LR, -LR and DOR of Xpert Xpress Flu/RSV to detect Flu A were 0.96 (95\% Cl: 0.95, 0.98), 0.97 (95\% Cl: 0.96, 0.97), 80.65 (95\% Cl: $32.02,203.16), 0.02$ (95\% Cl: 0.01, 0.08) and 4806.90 (95\% Cl: 907.93, 25449.35), respectively. The SROC curves in Flu A are shown in Figure 2F. AUC was 0.9984, and the Q index was 0.9866 (SE = 0.0047). No publication bias was found in Deek's funnel plot of Flu A (Figure $2 G$ ).. In addition, the Egger test indicated that the publication bias of these articles in Flu A was low $(P=0.633)$.

\section{Influenza B}

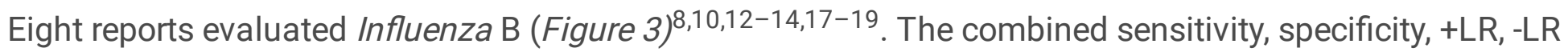
and DOR of Xpert Xpress Flu/RSV to detect Flu B were 0.98 (95\% Cl: 0.96, 0.99), 0.99 (95\% Cl: 0.99, 1.00), 163.74 (95\% Cl: 107.43, 249.55), 0.03 (95\% Cl: 0.01, 0.06) and 6266.78 (95\% Cl: 2642.01, 14864.68), respectively. The SROC curves in Flu A are shown in Figure 3F; AUC was 0.9990 and the Q index was 0.9912 (SE = 0.0030). No publication bias was found in Deek's funnel plot of Flu B (Figure 3G).. Furthermore, the Egger test indicated that the publication bias of these articles in Flu $B$ was low $(P=$ $0.353)$.

\section{Respiratory syncytial virus (RSV)}


Five reports evaluated the RSV (Figure 4) $10,12,14,17,18$. The combined sensitivity, specificity, +LR, -LR and DOR of Xpert Xpress Flu/RSV to detect RSV were 0.96 (95\% Cl: 0.93, 0.98), 1.00 (95\% Cl: 0.99, 1.00), 237.35 (95\% Cl: 137.80, 408.82), 0.05 (95\% Cl: 0.02, 0.04) and 5254.41 (95\% Cl: 2229.31, 12384.47), respectively. The SROC curves in Flu A are shown in Figure 4F; AUC $=0.9993$, and the Q index was 0.9947 $(S E=0.0028)$. No publication bias was found in Deek's funnel plot of RSV (Figure $4 G)$.. Moreover, the Egger test indicated that the publication bias of these articles in RSV was low $(P=0.584)$.

\section{Discussion}

Flu and RSV are two common pathogens that cause respiratory tract infections. Thus, developing new tools for rapid diagnosis of Flu or RSV is of extreme importance. In this study, we extracted 22 groups of data from nine articles that evaluated the diagnostic accuracy of Xpert Xpress Flu/RSV for detection of Flu and RSV performance from inception to April 2019, including 4626 Influenza A samples, 4414 Influenza B samples, and 3662 RSV samples. We analyzed the articles to verify the accuracy of Xpert Xpress Flu/RSV for detecting Flu A, Flu B, and RSV. The results of the study showed that the combined sensitivity of Xpert Xpress Flu/RSV in the diagnosis of Flu A, Flu B and RSV was respectively $0.96,0.98$, 0.98 ; the combined specificity was $0.97,0.99,0.99$; +LR was $80.65,163.74,237.85$; - LR was $0.02,0.03$, 0.05; DOR was 4806.90, 6266.78, 5254.41; and the SROC AUC was 0.9984, 0.9990, 0.9993. Among them, the + LR of Flu A, Flu B, and RSV were all larger than 10, while -LR were both $<0.1$. For Flu A, a pooled $+L R$ of 80.65 and a pooled -LR of 0.02 showed that patients with positive or negative Xpert were 80.65 times more likely to develop Flu A or 0.02 times less likely to develop Flu A. The SROC AUC of Flu A, Flu B, and RSV were all very close to 1 and the SROC curve was close to the upper left corner; the area under the curve was extremely large. AUC data (all over 0.9 ) indicated that the overall diagnostic accuracy of Xpert for Flu and RSV was relatively high. In addition, the DOR of Flu A, Flu B, and RSV were very large, indicating that the correct diagnosis was far greater than the wrong diagnosis. To sum up, Xpert Xpress Flu/RSV is highly accurate for the diagnosis of Flu A, Flu B and RSV. Moreover, the bias coefficients of Flu $A$, Flu $B$, and RSV were $P=0.633,0.353,0.584$; since these were all greater than 0.05 , it is possible to conclude that there is a subtle publishing bias.

PCR is a gold standard approach or the detection of Flu and RSV. PCR requires small amount of DNA fragments and possesses high sensitivity and specificity for detection. The disadvantages of traditional PCR compared to Xpert Xpress Flu/RSV are the following: first, traditional PCR requires longer time to be completed (e.g. traditional RT-PCR takes about 3-4 hours to interpret all the data manually). Xpert Xpress Flu/RSV can be performed in 30 minutes, which helps clinicians to make timely diagnosis and improve patients' satisfaction ${ }^{21}$. Secondly, the traditional RT-PCR requires skilled workers, expensive equipment, and has high requirement for sample collection, storage, and processing; while all steps in Xpert Xpress Flu/RSV assay, including sample extraction, nucleic acid purification, and concentration, quantitative PCR amplification detection and analysis are completed automatically, which in turn significantly decreases the possibility of errors and time for diagnosis ${ }^{22}$. In addition, we demonstrated that Xpert Xpress Flu/RSV 
has high sensitivity and specificity. In summary, Xpert Xpress Flu/RSV is more suitable for clinical application.

This current study has several limitations: first, the sensitivity and specificity of Flu and RSV were calculated only on the basis of all the samples included in the articles. However, when applied in the clinic, they need to be considered more carefully in combination with the clinical situation. Applications of Xpert for Flu and RSV cases have been reported in some studies; however, the data are very limited. More data are needed in the future to supplement the results of diagnosis of Flu and RSV by Xpert Xpress Flu/RSV. Secondly, we did not conducted group evaluation because of the limited literature, small sample size and lack of comparability.

\section{Conclusions}

Xpert Xpress Flu/RSV is a valuable method for detection of Flu and RSV in vitro, with high sensitivity and specificity. However, further research is necessary to determine whether Xpert Xpress Flu/RSV could be used as a clinical diagnostic standard for the identification of Flu and RSV.

\section{Abbreviations}

FluA: Influenza A; FluB: Influenza B; RSV: Respiratory syncytial virus; QUADAS-2: Quality Assessment of Diagnostic Accuracy Studies; +LR: Positive likelihood ratio; -LR: Negative likelihood ratio; DOR: Diagnostic ratio; SROC: Summary receiver operating characteristic curves; AUC: Area under the curve; Cl: Confidence intervals; PCR: Polymerase chain reaction; ARDS: Acute respiratory distress development syndrome; FDA: the US Food and Drug Administration; EMA: European Medicines Agency; NMPA: National Medical Products Administration

\section{Declarations}

\section{Ethics approval and consent to participate}

Not applicable.

\section{Consent for publication}

Not applicable.

\section{Availability of data and materials}

All data generated or analyzed during this study are included in this published article and its supplementary information files. 


\section{Competing interests}

The authors declare that they have no competing interests.

\section{Funding}

No funding was used to support this study.

\section{Authors' contributions}

XG planned and designed the experiments. YL, TX, WD and GL retrieved and reviewed the study reports, summarized and analysed the data. QL contributed to the application of the analysis tools. All authors participated in the writing, reading, and revising of the manuscript and approved the final manuscript.

\section{Acknowledgements}

Not applicable.

\section{References}

1Peteranderl, C., Herold, S. \& Schmoldt, C. Human Influenza Virus Infections. Seminars in respiratory and critical care medicine 37, 487-500, doi:10.1055/s-0036-1584801 (2016).

2Liu, X. X. et al. Excess mortality associated with influenza after the $2009 \mathrm{H} 1 \mathrm{~N} 1$ pandemic in a subtropical city in China, 2010-2015. Int J Infect Dis 57, 54-60, doi:10.1016/j.ijid.2017.01.039 (2017).

3Jorquera, P. A., Anderson, L. \& Tripp, R. A. Human Respiratory Syncytial Virus: An Introduction. Methods in molecular biology (Clifton, N. J.) 1442, 1-12, doi:10.1007/978-1-4939-3687-8_1 (2016).

4Habibi, M. S. \& Chiu, C. Controlled human infection with RSV: The opportunities of experimental challenge. Vaccine 35, 489-495, doi:10.1016/j.vaccine.2016.08.086 (2017).

5Shi, T. et al. Global, regional, and national disease burden estimates of acute lower respiratory infections due to respiratory syncytial virus in young children in 2015: a systematic review and modelling study. Lancet (London, England) 390, 946-958, doi:10.1016/s0140-6736(17)30938-8 (2017).

6 Wouters, Y. et al. Comparison of the Idylla Respiratory (IFV-RSV) panel with the GeneXpert Xpert Flu/RSV assay: a retrospective study with nasopharyngeal and midturbinate samples. Diagnostic microbiology and infectious disease 94, 33-37, doi:10.1016/j.diagmicrobio.2018.11.022 (2019). 
7Caliendo, A.M. Multiplex PCR and emerging technologies for the detection of respiratory pathogens. Clinical infectious diseases: an official publication of the Infectious Diseases Society of America 52 Suppl 4, S326-330, doi:10.1093/cid/cir047 (2011).

8Schmidt, R. L. J. et al. A novel PCR-based point-of-care method facilitates rapid, efficient, and sensitive diagnosis of influenza virus infection. Clinical microbiology and infection: the official publication of the European Society of Clinical Microbiology and Infectious Diseases 25, 1032-1037, doi:10.1016/j.cmi.2018.12.017 (2019).

9Stellrecht, K. A. et al. Effect of genomic drift of influenza PCR tests. Journal of clinical virology: the official publication of the Pan American Society for Clinical Virology 93, 25-29, doi:10.1016/j.jcv.2017.05.016 (2017).

10Zou, X. et al. Comparison of the Cepheid Xpert Xpress Flu/RSV assay and commercial real-time PCR for the detection of influenza A and influenza B in a prospective cohort from China. Int J Infect Dis 80 , 92-97, doi:10.1016/j.jijid.2018.12.014 (2019).

11DiMaio, M. A., Sahoo, M. K., Waggoner, J. \& Pinsky, B. A. Comparison of Xpert Flu rapid nucleic acid testing with rapid antigen testing for the diagnosis of influenza A and B. Journal of virological methods 186, 137-140, doi:10.1016/j.jviromet.2012.07.023 (2012).

12Cohen, D. M. et al. Accurate PCR Detection of Influenza A/B and Respiratory Syncytial Viruses by Use of Cepheid Xpert Flu+RSV Xpress Assay in Point-of-Care Settings: Comparison to Prodesse ProFlu. Journal of clinical microbiology 56, doi:10.1128/jcm.01237-17 (2018).

13Chen, J. H. et al. Evaluation of the molecular Xpert Xpress Flu/RSV assay vs. Alere i Influenza A \& B assay for rapid detection of influenza viruses. Diagnostic Microbiology and Infectious Disease 90, 177180, doi:10.1016/j.diagmicrobio.2017.11.010 (2018).

14Popowitch, E. B. \& Miller, M. B. Comparison of the Xpert Flu/RSV XC and Xpress Flu/RSV assays. Journal of Clinical Microbiology 56, doi:10.1128/JCM.00278-18 (2018).

15Deville, W. L. et al. Conducting systematic reviews of diagnostic studies: didactic guidelines. $B M C$ medical research methodology 2, 9 (2002).

16Whiting, P. F. et al. QUADAS-2: a revised tool for the quality assessment of diagnostic accuracy studies. Annals of internal medicine 155, 529-536, doi:10.7326/0003-4819-155-8-201110180-00009 (2011).

17Banerjee, D., Kanwar, N., Hassan, F., Essmyer, C. \& Selvarangan, R. Comparison of six sample-to-answer influenza $A / B$ and respiratory syncytial virus nucleic acid amplification assays using respiratory specimens from children. Journal of Clinical Microbiology 56, doi:10.1128/JCM.00930-18 (2018). 
18Ling, L. et al. Parallel validation of three molecular devices for simultaneous detection and identification of influenza A and B and respiratory syncytial viruses. Journal of Clinical Microbiology 56, doi:10.1128/JCM.01691-17 (2018).

19 Valentin, T. et al. Prospective evaluation of three rapid molecular tests for seasonal influenza in patients presenting at an emergency unit. Journal of clinical virology: the official publication of the Pan American Society for Clinical Virology 111, 29-32, doi:10.1016/j.jcv.2019.01.003 (2019).

20To, K. K. W. et al. Saliva as a diagnostic specimen for testing respiratory virus by a point-of-care molecular assay: a diagnostic validity study. Clinical microbiology and infection: the official publication of the European Society of Clinical Microbiology and Infectious Diseases 25, 372-378, doi:10.1016/j.cmi.2018.06.009 (2019).

21Cai, Z. H., Dai, Y. Y., Huang, L. Y., Zhang, W. S. \& Guo, X. G. Diagnosis of mycoplasma pneumoniae by loop-mediated isothermal amplification: systematic review and meta-analysis. BMC infectious diseases 19, 173, doi:10.1186/s12879-019-3799-4 (2019).

22Cepheid Xpert Xpress Flu/RSV productcatalog, <https://www.cepheid.com/administrator/components/com_productcatalog/libraryfiles/d6c1bb5aeba2735a7a547c598a277627-Xpert-Xpress-Flu-Brochure-US-0618-02.pdf> (

\section{Tables}

Due to technical limitations, tables are only available as a download in the supplemental files section

\section{Figures}



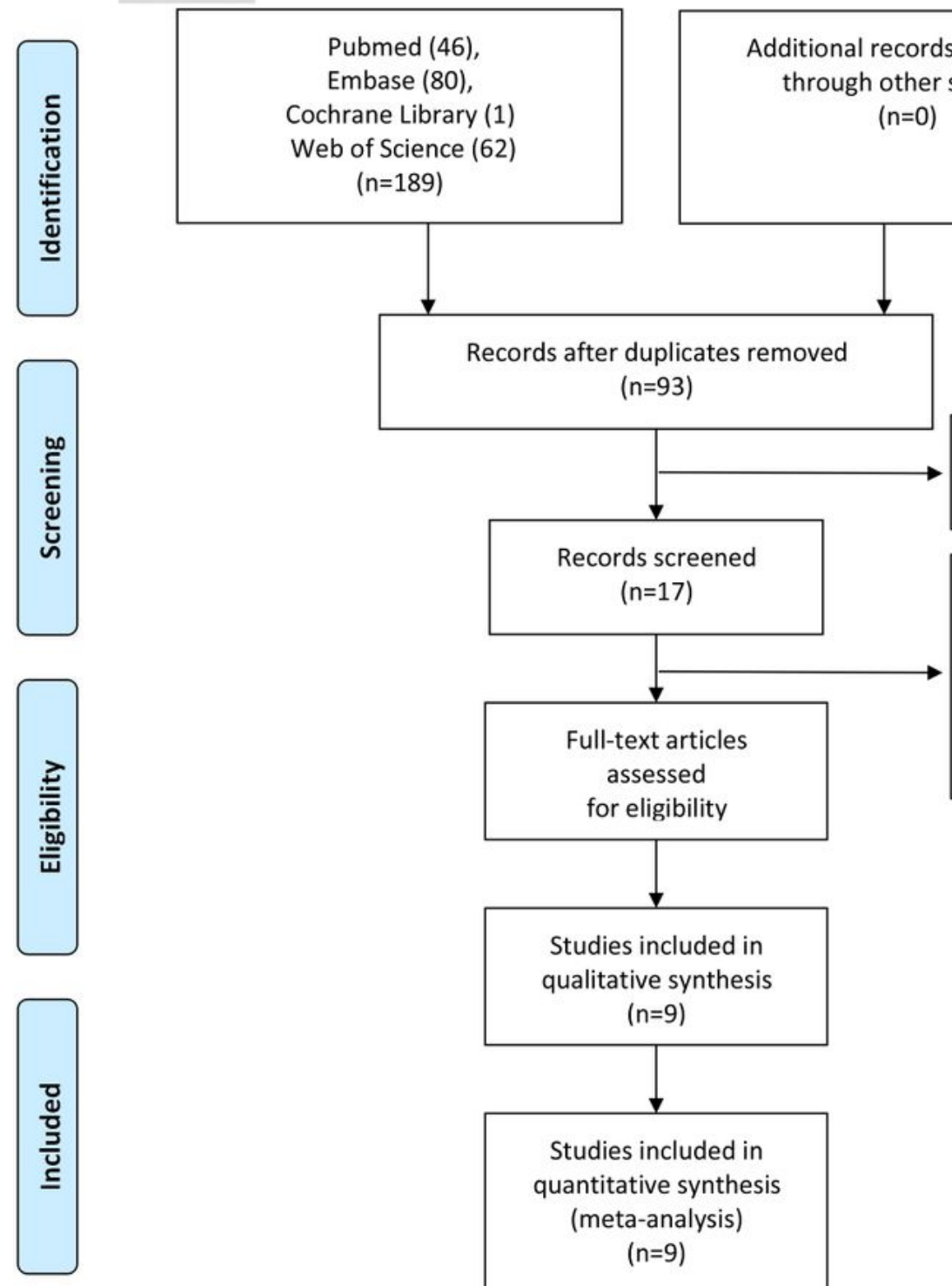

76 excluded by screening title/abstract

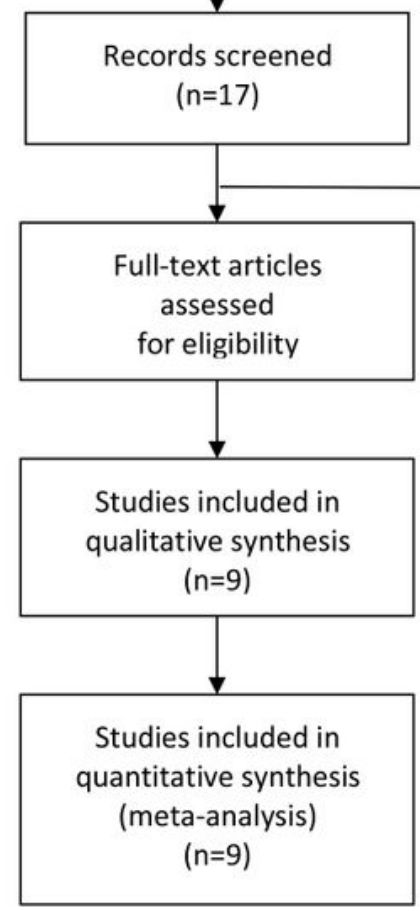

8 articles excluded after reading full text Reasons for exclusion: Conference abstract(5) Insufficient information(2) Letter(1)

From: Moher D, Liberati A, Tetzlaff J, Altman DG, The PRISMA Group (2009). Preferred Reporting /tems for Systematic Reviews and MetaAnalyses: The PRISMA Statement. PLoS Med 6(6): e1000097. doi:10.1371/journal.pmed1000097

For more information, visit www.prisma-statement.org.

\section{Figure 1}

PRISMA flow diagram of publications searched.

\section{Figure 2}


Forest plots of (A) the combined sensitivity, (B) specificity, (C) positive LR, negative LR, (D) diagnostic OR, $(F)$ the summary receiver operating characteristic (SROC) curve of Xpert Xpress Flu/RSV for the diagnosis of Influenza A and Deeks' funnel plot asymmetry test to assess publication bias for Xpert Xpress Flu/RSV detection of Influenza A (G).
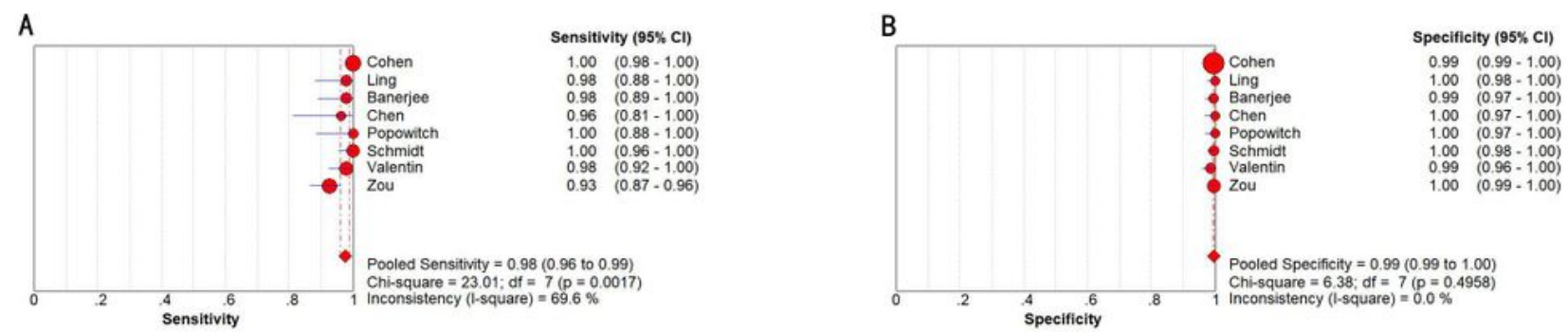

C

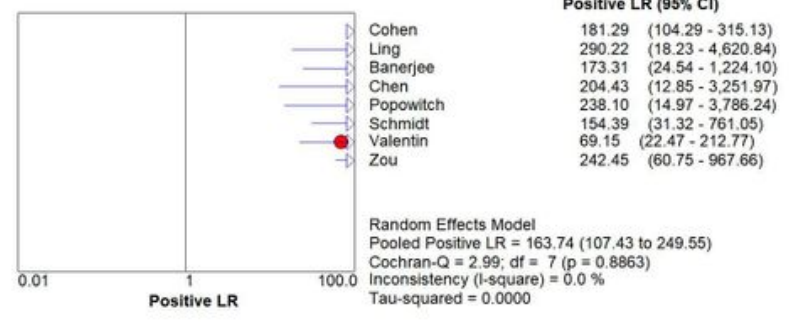

Positive LR $(95 \% \mathrm{Cl})$ $181.29 \quad(104.29-315.13)$ $\begin{array}{ll}290.22 & (18.23-4.620 .84) \\ 173.31 & (24.54-1,224.10)\end{array}$ $204.43 \quad(12.85-3.251 .97)$ $(14.97-3.786 .24)$ $154.39(31.32-761.05)$ $\begin{array}{rr}9.15 & (22.47-212.77) \\ 242.45 & (60.75-967.66)\end{array}$ $=0.0 \%$
D

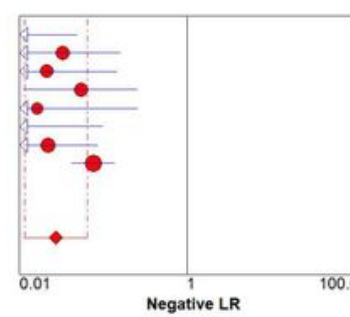

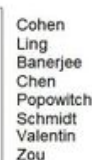

$\begin{array}{ll}0.00 & (0.00-0.05) \\ 0.03 & (0.01-0.16)\end{array}$

$0.02(0.00-0.15)$

$0.02 \quad 0.000 .025$

$0.07 \quad(0.04-0.14)$

Random Effects Model

Pooled Negative $L R=0.03(0.01$ to 0.06$)$ Cochran- $Q=15.60 ; \mathrm{df}=7 \quad(\mathrm{p}=0.0290)$

Tau-squared $=0.7400$

\section{Figure 3}


Forest plots of (A) the combined sensitivity, (B) specificity, (C) positive LR, negative LR, (D) diagnostic OR, $(F)$ the summary receiver operating characteristic (SROC) curve of Xpert Xpress Flu/RSV for the diagnosis of Influenza B and Deeks' funnel plot asymmetry test to assess publication bias for Xpert Xpress Flu/RSV detection of Influenza B (G).
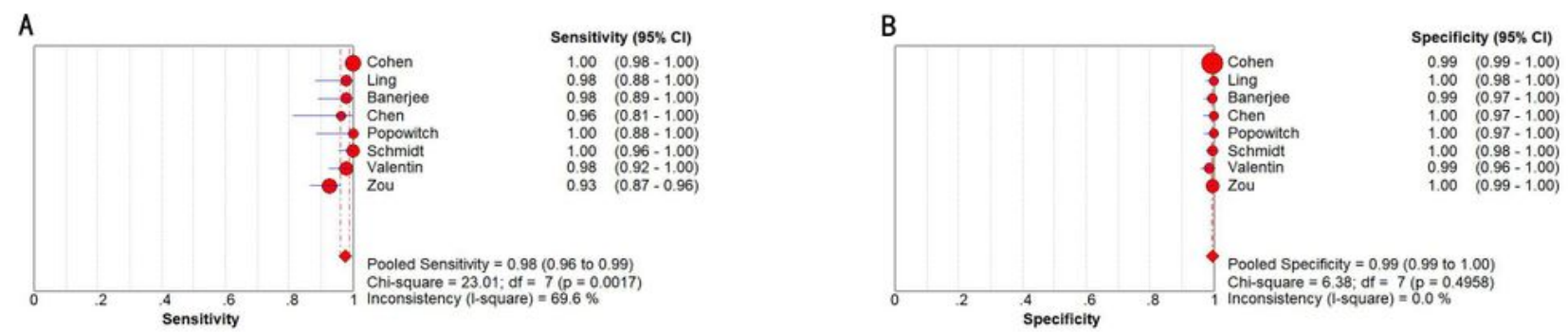

C
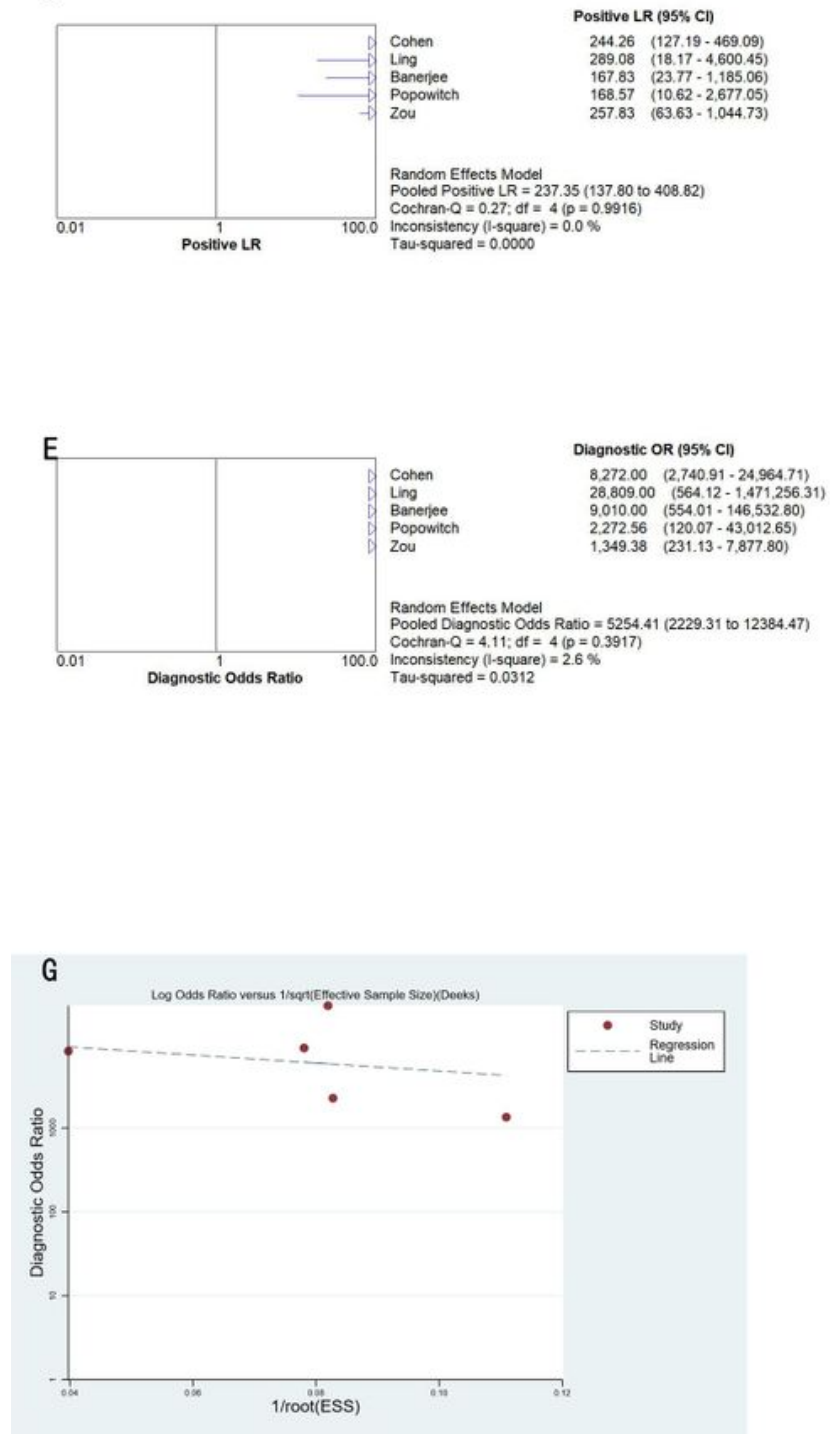

D
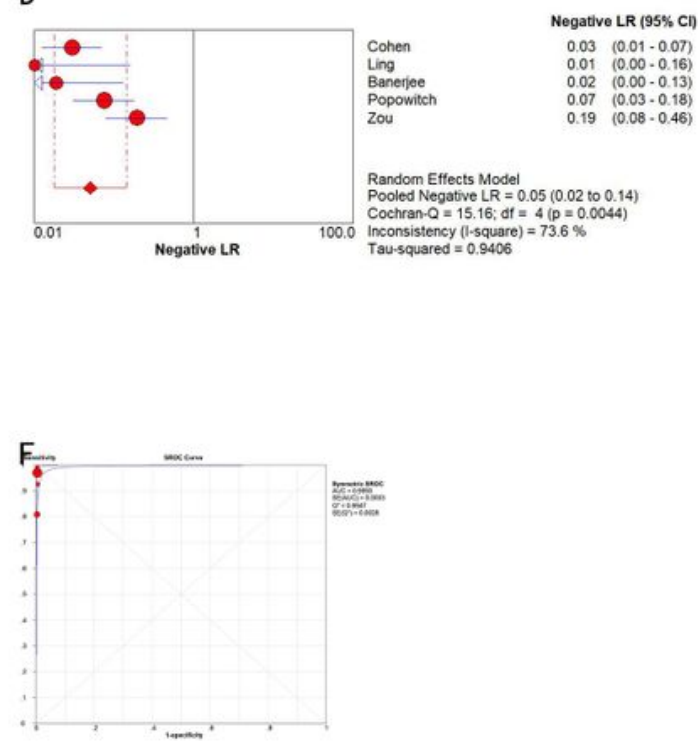

Figure 4 
Forest plots of (A) the combined sensitivity, (B) specificity, (C) positive LR, negative $L R,(D)$ diagnostic OR, $(F)$ the summary receiver operating characteristic (SROC) curve of Xpert Xpress Flu/RSV for the diagnosis of RSV and Deeks' funnel plot asymmetry test to assess publication bias for Xpert Xpress Flu/RSV detection of RSV (G).

\section{Supplementary Files}

This is a list of supplementary files associated with this preprint. Click to download.

- Table1.Characteristicsoftheincludedstudies.doc

- Table2.Qualityevaluationoftheincludedstudies..docx 